Meeting report

\title{
Use of the Herpes-simplex-1-Thymidine Kinase gene as a therapeutic tool in allogeneic
} hemapoietic stem cell transplantation

P. Tiberghien, J.Y. Cahn, C. Ferrand, N. Milpied, E. Contassot, R. Angonin, D. Sallard, E. Robinet, C.W. Reynolds, J.M. Certoux, E. Rousseau, W. Jacob and P. Hervé

ETS de Franche-Comté, Services d'Hématologie et d'Anatomie-Pathologie, CHU Besançon, France; Service d'Hématologie, CHU Nantes, France; NCI-FCRDC, Frederick, Genetic Therapy, Inc, Gaithersburg, Maryland, USA Correspondence to: $\mathrm{P}$. Tiberghien

Ex vivo T-cell graft prevents graft-versus-host disease (GvHD) after allogeneic hematopoietic stem cell transplantation but results in an increase in graft rejection and a reduction in the graft-versus-leukemia effect. The use of donor T-cells expressing herpes-simplex thymidine-kinase (HS-tk) gene could allow, if GVHD was to occur, a selective in vivo depletion of these T-cells by the use of ganciclovir (GCV). On the other hand, patients not suffering from GvHD could continue to benefit from the favorable effects of these Tcells on engraftment and tumor control. We have previously established that retroviral-mediated transfer of the HS-tk and neomycine-resistance genes in primary T-lymphocytes, followed by G418 selection, resulted in T-cells maintaining IL-2- and allo-responsiveness and specifically inhibited by GCV [Blood 84 : 1333].

The ex vivo manipulation of the donor T-lymphocytes requires a 12 day culture period, including polyclonal stimulation (OKT-3, II-2), retroviral transduction and G418 selection. Murine studies performed in our laboratory demonstrated that T-lymphocytes cultured in vitro for 12 days were still capable of mediating strong alloreactivity in vivo. Further studies involving HS-tk-expressing transgenic mice (in collaboration with David Klatzmann and coworkers) are presently underway.

In order to determine the optimal gene transfer protocol, we evaluated the influence of the following variables on transduction efficiency (prior to G418 selection): number of retroviral exposures (1 vs 2), incubation temperature $\left(37 \mathrm{vs} 32^{\circ} \mathrm{C}\right)$, spin transduction, use of culture bags versus flasks. No significant differences were found between the different transduction protocols. The most important variable was the donor with similar transduction efficiencies within each donor, whatever the transduction process used.

We have recently initiated a phase I study evaluating the feasibility and toxicity of administrating HS-tkexpressing donor T-cells in conjunction with a T-cell depleted marrow graft. Based on the findings described above, as well as practical and safety considerations, the transduction protocol used in our trial is a single $24 \mathrm{~h}$ incubation at $37^{\circ} \mathrm{C}$. All the steps of cell preparation from initial cell harvest from the donor to infusion of the gene-modified cells in the patient are performed in closed system involving the use of bags and a sterile connecting device. Three patients at high risk of GvHD (recipients over 40 years old and/or sex-mismatch female into male) have received $2.10^{5}$ genetically modified T-lymphocytes/kg in conjunction with a T-cell depleted marrow after an intensified conditioning regimen (cytoxan, thiotepa and total body irradiation).

By hopefully establishing the feasibility of such an approach, our study should contribute to development of gene transfer as a mean of modulating allogeneic reactivity after hematopoietic stem cell transplantation.

Back to the HCT-EE Home Page

Last change: April 28, 1997

helpdesk.link@springer.de

(C) 1996 by Springer-Verlag France 